\title{
Formula optimization of Cordyceps Flower beverage based on sensory analysis experiments
}

\author{
Hui-hui $\mathrm{Li}^{1}$, Rong Huang ${ }^{1}$, Ming-chen $\mathrm{Li}^{2}{ }^{*}$ \\ ${ }^{1}$ College of Food Technology, Wuhan Business University, Wuhan, China \\ ${ }^{2}$ Vegetarian Institute of Wuhan, Wuhan Business University, Wuhan, China
}

\begin{abstract}
Cordyceps flower is a new resource as a food and drug, which has a broad market prospect. The paper using Cordyceps flower sporocarp as raw material and adopting sensory results as evaluation criteria, through single factor and orthogonal experiments optimize cordyceps flower beverage formula. The results show that Cordyceps powder should be first leached in a water bath at $80^{\circ} \mathrm{C}$ for $15 \mathrm{~min}$ at a feed-liquid ratio of 1:30. After centrifugation and filtration, cordyceps flower liquid was obtained. Then the liquid should be reconstitute into $30 \%$ diluent. Third, adding $0.04 \%$ aspartame, $0.06 \%$ citric acid and $0.1 \%$ sodium carboxyl methyl cellulose $(\mathrm{CMC})$, a tasty and nutritious cordyceps flower beverage was developed ultimately.
\end{abstract}

\section{Introduction}

The scientific name for Cordyceps Flower is Cordyceps militaris (Fr.) Link, which had been classified as a a new resource food by the Ministry of Health in 2009. Cordyceps Flower in market is artificially cultured Cordyceps fruiting body. It usually reaches a length of 2-8 $\mathrm{cm}$ while the appearance mostly takes on the form of orange grass. Cordyceps flower is not only rich in protein and vitamins, but also contains bioactive substances such as cordycepin, cordyceps polysaccharide, cordycepic acid, ergosterol, SOD and zeaxanthin[1], which has broad market prospect. A batch of health food with cordyceps flower has emerged on the market,especially cordyceps flower beverage. There are two main production raw material of cordyceps flower beverage. One is the fermentation broth of Cordyceps Flower hyphae, the other is extracting solution of Cordyceps fruiting body[1]. Since Cordyceps Flower has a unique taste, the majority of Cordyceps Flower drinks are compound drinks with another kind of health function raw materials, such as Jujuba dates [2], wolfberry [3], ginkgo biloba [4], ganoderma lucidum [5], mulberry leaves [6], hawthorn [7] while researches on beverage of single Cordyceps Flower is relatively rare [8]. Therefore, the development of a beverage with Cordyceps Flowers as the main raw material can fill the market gap and enrich the beverage varieties. Furthermore it also can promote the utilization of cordyceps resources.

\section{Materials and Methods}

\subsection{Materials and instruments}

Materials: Cordyceps fruiting body artificially cultured by College of Food Science and Technology in Wuhan Business University、Watsons pure water.

Experiment reagents: Citric acid, analytically pure, Sinopharm Group Chemical Reagent Co. LTD; Aspartame and carboxyl methyl cellulose, food grade, Shandong Fufeng Fermentation Co. LTD.

Experiment instruments: YB-1500A high speed multifunction crusher, produced by Yongkang Sufeng Industry and Trade Co., Ltd.; stainless steel sieve (100 mesh), produced by Zhejiang Shangyu Daoxu Zhangxing Yarn Screen Factory; XS204 electronic balance, produced by Mettler Toledo Technology Instrument Co., Ltd.; BH-002 digital display constant temperature air dry oven, produced by Shanghai Yiheng Scientific Instrument Co., Ltd.; HH-6 thermostat water bath, produced by Guohua Electric Appliance Co., Ltd.; UV-1800 ultraviolet-visible spectrophotometer, produced by Shanghai Aoxi Scientific Instrument Co., Ltd.; TGL-16M high Speed Centrifuge, produced by Shanghai Luxiangyi Centrifuge Instrument Co., Ltd.; LX-B75L Vertical High-Pressure Steam Sterilizer, produced by Hefei Huatai medical facility Co., Ltd.; PSH-3 pH meter, produced by Shanghai Yidian Scientific Instrument Co., Ltd.

\footnotetext{
* Corresponding author: 502416671@qq.com
} 


\subsection{Experiment methods}

\subsubsection{Preparation of Cordyceps Flower beverage}

The production process of Cordyceps Flower beverage used in this paper was as follows: extracting Cordyceps Flower solution $\rightarrow$ blending $\rightarrow$ sterilizing $\rightarrow$ cooling $\rightarrow$ canning.

The optimum extraction process of Cordyceps Flower solution was obtained from the previous experiments. After quantitatively weighing the dry Cordyceps fruiting body, the fruiting body were crushed in a tissue crusher. Then added the powder sieved through 100 mesh to distilled water according to the ratio of $1: 30$. After leaching at $80^{\circ} \mathrm{C}$ water bath for $15 \mathrm{~min}$, the supernatant was centrifuged at $6000 \mathrm{r} / \mathrm{min}$ for $10 \mathrm{~min}$ at $4^{\circ} \mathrm{C}$. In this paper, the original solution of Cordyceps Flower was diluted with different concentrations, then mixed different sweeteners, acid agents and thickeners. After sterilizing at $121^{\circ} \mathrm{C}$ for 15 minutes and natural cooling, the Cordyceps Flower beverage was canned.

\subsubsection{Single factor experiment of formula optimization of Cordyceps Flower beverage}

Because of the higher concentration of Cordyceps Flower original solution, the sensory characteristic including color, taste and flavor are not acceptable, it needs to be diluted. In this experiment, the concentration of the original solution was $100 \%$, and the dilution was carried out according to $20 \%, 30 \%, 40 \%, 50 \%$ and $60 \%$ of the original solution. The appropriate concentration was selected through sensory analysis experiments.

After aspartame was selected as the sweetener in Cordyceps Flower beverage, according to the relevant literature [3,7], aspartame added amount was optimized. The maximum amount of aspartame was allowed to be $0.6 \mathrm{~g} / \mathrm{kg}$ in GB2760, so in the experiment the amount of aspartame was set to $0.01 \%, 0.02 \%, 0.03 \%, 0.04 \%$ and $0.05 \%$ (mass score) respectively. The optimal amount of aspartame is selected based on sensory evaluation.

Citric acid was determined as acidity agent in preliminary experiment. Then 5 concentration levels were set in the experiment referring to relevant literature [6,8]: $0.06 \%, 0.08 \%, 0.10 \%, 0.12 \%$ and $0.14 \%$. The optimal amount of citric acid is determined by sensory evaluation.

According to related research[9,10], CMC was selected as the sweetener in Cordyceps Flower beverage. The amount of CMC is optimized from $0.01 \%, 0.02 \%$, $0.03 \%, 0.04 \%$ and $0.05 \%$ (mass score) based on sensory evaluation.

\subsubsection{The orthogonal experiment of Cordyceps Flower beverage}

Based on the result of single factor experiment of Cordyceps Flower beverage, "concentration of concentration of Aspartame, Citric acid,CMC and diluent concentration of Cordyceps Flower original solution" these four factors and different levels was chose to carry out nine sets of orthogonal experiment (as shown in Table 1). And each set were carried out three times in parallel.

Table1 Orthogonal experiment factors and levels table of Cordyceps Flower Beverage

\begin{tabular}{|c|c|c|c|c|}
\hline & $\begin{array}{c}\text { Extracting } \\
\text { solution (\%) }\end{array}$ & $\begin{array}{c}\text { Aspartame } \\
(\%)\end{array}$ & $\begin{array}{c}\text { Citric acid } \\
(\%)\end{array}$ & $\begin{array}{c}\text { CMC } \\
(\%)\end{array}$ \\
\hline 1 & 30 & 0.02 & 0.06 & 0.06 \\
\hline 2 & 40 & 0.03 & 0.08 & 0.08 \\
\hline 3 & 50 & 0.04 & 0.1 & 0.1 \\
\hline
\end{tabular}

\subsubsection{Sensory evaluation experiment of Cordyceps Flower beverage}

Ten experienced sensory evaluators were selected in this experiment. Three-digit random number coding was used to score the single factor experimental samples and orthogonal experimental samples. Sensory scores were made according to Table 2. Sensory evaluation was conducted on a 10-point scale and the total score was recorded. The weight of each evaluation index was mainly referred to literature [2,9] and adjusted according to the pre-experiment.

Table2 Sensory evaluation creterion of Cordyceps Flower Beverage

\begin{tabular}{|c|c|c|}
\hline Index & Evaluation criterion & Score \\
\hline \multirow{3}{*}{$\begin{array}{l}\text { Texture } \\
(2.0)\end{array}$} & $\begin{array}{l}\text { Clear and bright, } \\
\text { without precipitation }\end{array}$ & $1.5-2.0$ \\
\hline & $\begin{array}{l}\text { Clear and bright, } \\
\text { slightly precipitated }\end{array}$ & $1.0-1.5$ \\
\hline & Muddy, with obvious precipitation & $0.0-1.0$ \\
\hline \multirow{3}{*}{$\begin{array}{r}\text { Colour } \\
(2.0)\end{array}$} & Yellow & $1.5-2.0$ \\
\hline & Yellowish-brown & $1.0-1.5$ \\
\hline & Brown & $0.0-1.0$ \\
\hline \multirow{3}{*}{$\begin{array}{r}\text { Flavor } \\
(2.0)\end{array}$} & A light scent of cordyceps & $1.5-2.0$ \\
\hline & $\begin{array}{l}\text { A subtle or too thick scent of } \\
\text { cordyceps }\end{array}$ & $1.0-1.5$ \\
\hline & No scent of cordyceps or smelly & $0.0-1.0$ \\
\hline \multirow{3}{*}{$\begin{array}{l}\text { Taste } \\
(4.0)\end{array}$} & $\begin{array}{l}\text { Sour and sweet delicious, } \\
\text { pure taste, smooth taste }\end{array}$ & $3.0-4.0$ \\
\hline & Slightly sweet or sour, smooth taste & $2.0-3.0$ \\
\hline & Too sweet or too sour & $0.0-2.0$ \\
\hline
\end{tabular}

\subsubsection{Experimental data statistics}

Sensory evaluation score of single factor experiment and orthogonal experiments were calculated based on the average of ten parallel experiments. In the single factor experiment, Excel was used to analyze the mean value and standard deviation of sensory evaluation indexes while the range analysis was performed by orthogonal assistant II V3.1. The model settings do not consider interactions, but examine the main effects of various factors. 


\section{Results and analysis}

\subsection{Single factor experiment of formula optimization of Cordyceps Flower beverage}

\subsubsection{Influence of extraction diluent concentration on Cordyceps Flower beverage}

After the optimum extraction process of Cordyceps Flower solution, the original solution of Cordyceps Flower was obtained. The original solution diluted 10 times, the absorbance value A stabilized at 0.31 when the incident wavelength of $\lambda$ was $480 \mathrm{~nm}$ in the UV-Vis spectrophotometer. Different concentration of Cordyceps Flower beverage were carried out according to $20 \%, 30 \%$, $40 \%, 50 \%$ and $60 \%$ dilution. With the increase of the concentration of extract, the color of the beverage gradually became brown and dark, and the color score showed a decreasing trend. But in terms of flavor and taste index, sensory scores increased first and then decreased with the increase of extract concentration. In general, when the original solution of Cordyceps Flower was diluted at $40 \%$, the total score of sensory evaluation was the highest while the flavor and taste were relatively coordinated (Fig.1).

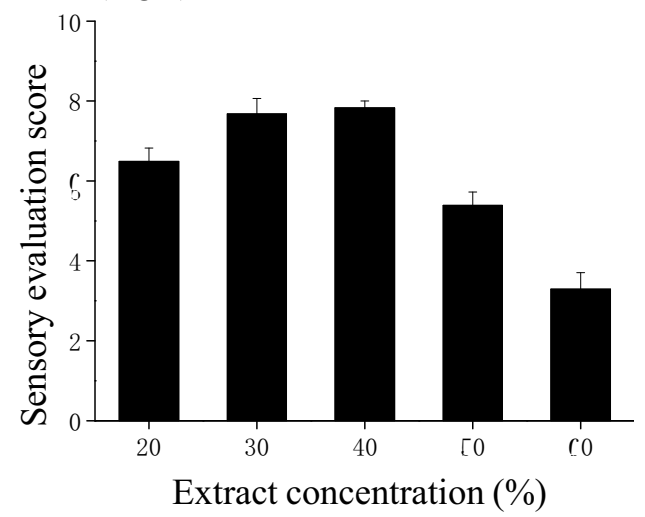

Fig.1 Influence of extraction dilution on sensory evaluation

\subsubsection{Influence of Aspartame on Cordyceps Flower beverage's Sensory evaluation}

In the pre-experiment, the sensory effects of sodium cyclamate, sucrose and aspartame on the cordyceps flower beverage were compared. The results showed that aspartame and sucrose were pure in sweetness while sodium cyclamate taste slow with slightly astringent. Aspartame is a combination product of aspartate, phenylalanine and methanol. It is not only pure in taste, but also high sweetness, economic benefits and low calories which is widely used in beverage processing [11]. As shown in Fig.2, when the concentration of aspartame increased from $0.01 \%$ to $0.06 \%$, sensory score showed a trend of first increase and then decrease. When its concentration was $0.03 \%$, the sensory score was the highest, and the cordyceps flower beverage reached a palatable sweetness.

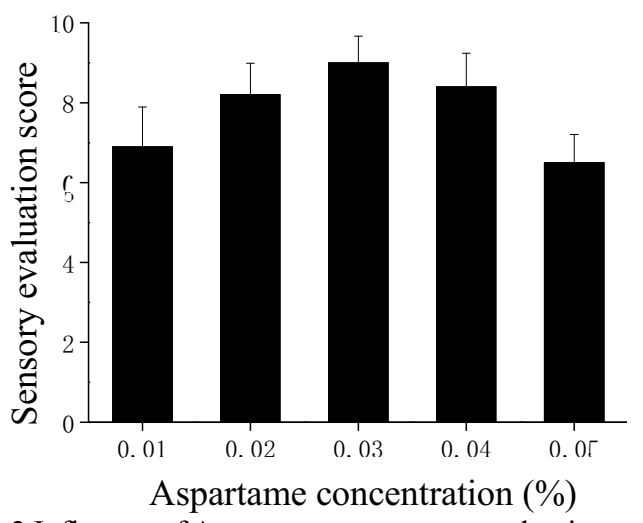

Fig.2 Influence of Aspartame on sensory evaluation

\subsubsection{Influence of Citric acid on Cordyceps Flower beverage's Sensory evaluation}

In the preliminary experiment, the sensory effects of ascorbic acid, tartaric acid and citric acid on cordyceps flower beverage were compared. Studies found that ascorbic acid as acid agent in Cordyceps Flower beverage tasted a bit irritant and uncomfortable while tartaric acid felt astringent.Only Citric acid made Cordyceps Flower beverage taste rounded and pleasant. According to Fig.3, when the concentration of citric acid was within the range of $0.06 \%-0.14 \%$, the total sensory score showed a trend of first increase and then decrease. The sensory reached the highest score when citric acid was $0.08 \%$ and at the same time Cordyceps flower beverage achieved more palatable acidity.

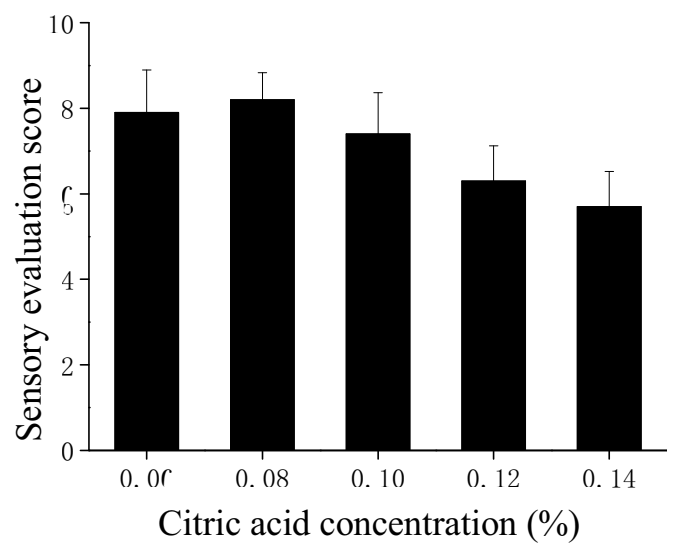

Fig.3 Influence of citric acid on sensory evaluation

\subsubsection{Influence of CMC on Cordyceps Flower beverage's Sensory evaluation}

Because the $\mathrm{pH}$ value of Cordyceps Flower original solution was 5.2, this acid unstable system was prone to delamination during storage. It is necessary to add a thickener in Cordyceps Flower beverage. Thickening agents were mostly hydrophilic polymer compounds, which could improve the smoothness and stability of the beverage. The sensory effects of CMC, guar gum and sodium alginate on Cordyceps Flower beverage were compared in the pre-experiment, and the results showed that $\mathrm{CMC}$ had the best effect. When the concentration of CMC increased from $0.06 \%$ to $0.14 \%$, the total sensory 
score of Cordyceps Flower beverage showed a trend of increasing first and then decreasing. When CMC concentration was $0.08 \%$, the sensory evaluation of the beverage was the best, with mellow taste and suitable viscosity (Fig.4). The effects of different thickeners on the storage life and stability of the beverage needed further study.

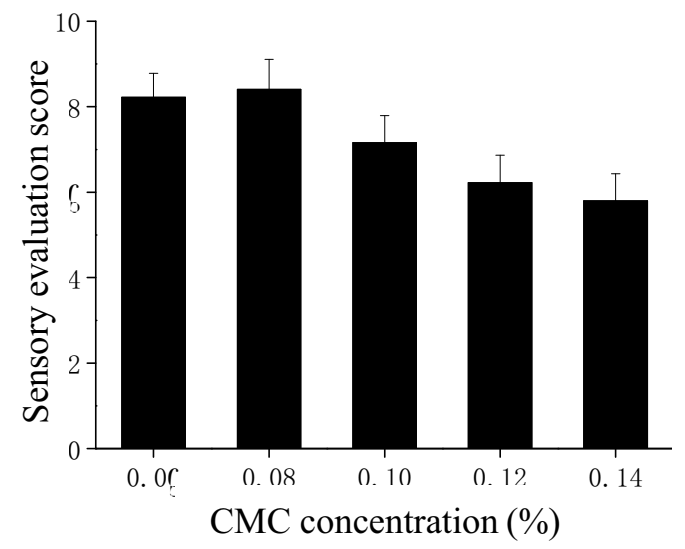

Fig.4 Influence of CMC on sensory evaluation

\subsection{Orthogonal experiment results and range analysis of Cordyceps Flower beverage}

According to the results of the single-factor experiment and the interaction between various factors, for example high concentration of Citric acid and CMC inhibited the sweetness of aspartame ${ }^{[11]}$, four factors such as "concentration of concentration of Aspartame, Citric acid,CMC and diluent concentration of Cordyceps Flower original solution" and respective three levels of respective factors were selected in $\mathrm{L}_{3}{ }^{4}$ Orthogonal experiment. The experimental results were showed that the optimal formula of the cordyceps flower beverage was $30 \%$ extract concentration, $0.04 \%$ aspartame concentration, $0.06 \%$ citric acid concentration and $0.1 \%$ CMC (Table 3.). In addition, it could be seen from the range analysis that the diluent concentration of Cordyceps Flower original solution had the greatest influence on the sensory effects of the beverage, followed by aspartame concentration, citric acid concentration, and $\mathrm{CMC}$ concentration.

Table3 Orthogonal experiment result of Cordyceps Flower Beverage

\begin{tabular}{|c|c|c|c|c|c|}
\hline & $\mathrm{A}$ & $\mathrm{B}$ & $\mathrm{C}$ & $\mathrm{D}$ & $\begin{array}{c}\text { Sensory } \\
\text { score }\end{array}$ \\
\hline Exp.1 & 30 & 0.02 & 0.06 & 0.06 & 7.65. \\
\hline Exp.2 & 30 & 0.03 & 0.08 & 0.08 & 8.11 \\
\hline Exp.3 & 30 & 0.04 & 0.1 & 0.1 & 7.93 \\
\hline Exp.4 & 40 & 0.02 & 0.08 & 0.1 & 6.96 \\
\hline Exp.5 & 40 & 0.03 & 0.1 & 0.06 & 6.31 \\
\hline Exp.6 & 40 & 0.04 & 0.06 & 0.08 & 8.40 \\
\hline Exp.7 & 50 & 0.02 & 0.1 & 0.08 & 5.28 \\
\hline Exp.8 & 50 & 0.03 & 0.06 & 0.1 & 7.11 \\
\hline Exp.9 & 50 & 0.04 & 0.08 & 0.06 & 7.23 \\
\hline Mean1 & 7.90 & 6.63 & 7.72 & 7.06 & \\
\hline Mean2 & 7.23 & 7.18 & 7.43 & 7.26 & \\
\hline
\end{tabular}

\begin{tabular}{|l|l|l|l|l|l|}
\hline Mean3 & 6.54 & 7.85 & 6.50 & 7.33 & \\
\hline Range & 1.36 & 1.22 & 1.22 & 0.27 & \\
\hline
\end{tabular}

Note:A: Extracting solution (\%), B: Aspartame $(\%)$, C: citric $\operatorname{acid}(\%), \mathrm{D}: \mathrm{CMC}(\%)$.

\section{Conclusion}

The fruitbody of Cordyceps Flower was used as raw material in this paper. Conbined with sensory evaluation technology, the technological formula of Cordyceps Flower beverage was optimized by single factor and orthogonal experiment. The results showed that with a material to liquid ratio of 1:30, the Cordyceps Flower powder was extracted in a water bath at $80^{\circ} \mathrm{C}$ for $15 \mathrm{~min}$. After centrifuge filtration, $30 \%$ diluent was prepared with $0.04 \%$ aspartame, $0.06 \%$ citric acid and $0.1 \% \mathrm{CMC}$. Then a palatable sweet and sour taste and nutrient-rich Cordyceps Flower beverage beverage was obtained. It was conducive to the utilization of Cordyceps Flower resources. A single sweetener, sour agent and thickener were used in Cordyceps Flower beverage in this paper while additives were often added mixed in beverage industry, so the preparation of Cordyceps Flower beverage needs to be further studied.

\section{Acknowlegement}

We acknowlege the financial support from General Programs of Natural Science Foundation in Science and Technology Department of Hubei Province (2019CFB764) and Vegetarian Institute of Wuhan.

\section{References}

1. J.H.Zhuo, X.Y.Gong, Y.M.Dong, et al. Food Sci. 5, 1-19 (2018)

2. S.S.Wang, S.Y.Zhou, X.R.Liu, et al. Preserv. Proc. 6, 33-38 (2017)

3. X.L.Qin, Y.M.Jin, L.Xing. Jiangsu Agric. Sci. 42, 227-229 (2014)

4. J.Z.Li, Y.Q.Huang, Z.W.Ye. Chin.brew. 34 (12), 175-178 (2015)

5. Y.Du. Shenyang Agric. Univ. [D]. 1-15 (2016)

6. J.Z.Li, Z.W.Ye, Y.Q.Huang. Food Res. Dev. 37, 77-79 (2016)

7. B.S.Lin, M.C.Luo. Food Sci. 34, 293-297 (2013)

8. M.Meng, J.Zhang, Z.Y.Zhu. Food Res. Dev. 37, 62-67 (2016)

9. C.Zhang, H.W.Chen. Agric. Eng. 2, 28-33 (2012)

10. Z.Y.Zhu, X.R.Liu, H.Q.Sun. J. Food Safety Quality Inspec. 7(7), 2914-2919 (2016)

11. X.Han. Zhejiang Ind. Bus. Univ. [D].1-23 (2013). 